\title{
A taxa de gestação em mulheres submetidas a técnicas de reprodução assistida é menor a partir dos 30 anos
}

\author{
The pregnancy rate in women submitted to assisted reproduction techniques is lower after the \\ age of 30 years
}

Lauriane Giselle de Abreu ${ }^{1}$, Laura Ferreira Santana ${ }^{1}$, Paula Andréa de Albuquerque Salles Navarro ${ }^{2}$, Rosana Maria dos Reis ${ }^{3}$, Rui Alberto Ferriani ${ }^{3}$, Marcos Dias de Moura ${ }^{3}$

\section{RESUMO}

Objetivo: avaliar as taxas de gestação diagnosticada química $(\beta-H C G>25 \mathrm{mUI} / \mathrm{mL}$ sérica obtida 14 dias após transferência embrionária) ou clinicamente (saco gestacional visualizado por ultra-sonografia quatro a seis semanas após transferência embrionária) e aborto em mulheres submetidas a fertilização in vitro (FIV) ou injeção intracitoplasmática de espermatozóide (ICSI) em serviço terciário de reprodução assistida e correlacionar estas taxas com a idade. Métodos: estudo transversal retrospectivo, com análise de 1016 ciclos de hiperestimulação ovariana controlada de 932 mulheres inférteis com indicação para FIV (370 ciclos) ou ICSI (646 ciclos). A idade das pacientes variou entre 22 e 46 anos. Todas as mulheres com idade superior a 35 anos incluídas no estudo apresentavam FSH < 15 UI/L. Os ciclos estudados foram divididos em dois grupos: o primeiro incluindo os referentes às mulheres com 22 a 30 anos e o segundo grupo, com aquelas dos 31 aos 46 anos. As variáveis analisadas foram: taxas de gravidez (química ou clínica) e aborto. O teste do $\chi^{2}$ foi empregado para comparar estas taxas entre os grupos. Resultados: a taxa total de gravidez foi 36,4\%, com redução significativa a partir dos 30 anos ( $\mathrm{p}=0,0001$ ). Dos 22 aos 30 anos (303 ciclos) a taxa de gestação foi de 45,4\%, enquanto que no grupo dos 31-46 anos (713 ciclos) foi de $25,1 \%$. As taxas de aborto foram, respectivamente, 10,2 e $11,6 \%$ $(\mathrm{p}=0,6854)$. Conclusão: apesar de não ter ocorrido diferença na taxa de aborto entre os grupos, observamos redução significativa na taxa de gestação de mulheres inférteis submetidas a FIV ou ICSI a partir dos 30 anos. Assim, recomendamos aos ginecologistas que não posterguem a investigação e o encaminhamento para tratamento da infertilidade.

PALAVRAS-CHAVE: Técnicas reprodutivas assistidas; Fertilidade; Oócitos; Fertilização in vitro; Injeções de esperma intracitoplasmáticas

\section{ABSTRACT}

Purpose: to evaluate the rate of chemically diagnosed pregnancy (serum $\beta$-HCG $>25 \mathrm{mIU} / \mathrm{mL}$ levels obtained fourteen days after the embryonic transfer) clinically diagnosed pregnancy (gestational sac visualized by ultrasound four to six weeks after the embryonic transfer) and miscarriage rates in women submitted in vitro fertilization to (IVF) and intracytoplasmic sperm injection (ICSI) in a tertiary center of assisted reproduction and to correlate these rates with age. Methods: retrospective transverse study with analysis of 1016 cycles of controlled ovarian hyperstimulation of 932 infertile women with indication of IVF ( 370 cycles) or ICSI ( 646 cycles). The patients' age ranged from 22 to 46 years. All women with age over 35 years included in the study had FSH<15 IU/L. The studied cycles were divided into two groups: the first including the cycles referring to women of 22 to 30 years and the second group, to those of 31 to 46 years. The studied variables were: pregnancy (chemical or clinical) and miscarriage rates. The $\chi^{2}$ test was used to compare these rates between the groups. Results: the total pregnancy rate was $36.42 \%$, with a significant reduction starting at 30 years ( $\mathrm{p}=0.0001)$. From 22 to 30 years ( 303 cycles) the pregnancy rate was $45.4 \%$, while in the range from 31 to 46 years (713 cycles), it was $25.1 \%$. The miscarriage rates were, $10.211,6 \%$, respectively $(\mathrm{p}=0.6854)$. Conclusion: although the miscarriage rates did not differ between the groups, a decrease in the pregnancy rate of infertile women submitted to IVF or ICSI was observed after the age of 30 years. Thus, we recommend the gynecologists not to postpone the investigation and the referral for treatment of infertility.

KEYWORDS: Assisted reproductive techniques; Fertility; Oocytes; In vitro fertilization; Sperm injections, intracytoplasmic

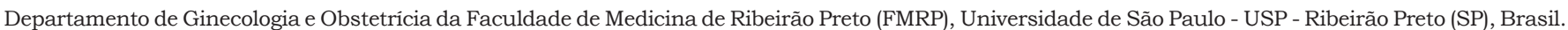

1 Pós-graduandas do Departamento de Ginecologia e Obstetrícia da FMRP, Universidade de São Paulo - USP - Ribeirão Preto (SP), Brasil.

2 Médica Assistente do Departamento de Ginecologia e Obstetrícia FMRP, Universidade de São Paulo - USP - Ribeirão Preto (SP), Brasil..

3 Docentes do Departamento de Ginecologia e Obstetrícia da FMRP, Universidade de São Paulo - USP - Ribeirão Preto (SP), Brasil.

Correspondência: Lauriane Giselle de Abreu

Laboratório de Ginecologia e Obstetrícia - $1^{\circ}$ andar - Hospital das Clínicas /Campus Universitário

Av. Bandeirantes n 3900 - 14049-900 - Ribeirão Preto - SP - Fone/fax: (16) 3602-2311 - e-mail: laurianegiselle@yahoo.com.br 
Introdução

Com o aumento da idade, a fecundidade natural e as taxas de gestação declinam mesmo em procedimentos de reprodução assistida ${ }^{1}$. A fertilidade feminina declina a partir dos 30 anos de ida$\mathrm{de}^{2-4} \mathrm{e}$, a partir dos 40 anos, há redução pela metade das taxas de gestação ${ }^{4}$. Durante a vida reprodutiva, tanto o número de oócitos se reduz rapidamente como a qualidade oocitária se altera ${ }^{1}$. Nas mulheres com mais de 40 anos, as aneuploidias são mais freqüentes, a taxa de aborto aumenta de 2 a 3 vezes e as taxas de implantação após fertilização in vitro (FIV) se reduzem ${ }^{1,4,5}$.

A pior qualidade oocitária parece ser o fator preponderante para esta redução da fertilidade observada com o avançar da idade, contrapondo outros fatores como a receptividade endometrial ${ }^{6}$. Esta afirmativa é reforçada por estudos em reprodução assistida usando programas de doação de oócitos, os quais mostraram que mulheres com mais de 40 anos que receberam oócitos de mulheres jovens apresentaram a mesma taxa de gestação clínica e de parto que as doadoras de oócitos ${ }^{6,7}$.

Em procedimentos de reprodução assistida, a qualidade oocitária é mais relevante do que a quantidade oocitária quando se consideram as taxas de sucesso ou taxa de gestação ${ }^{2,8}$. Porém, não se dispõe de nenhum método objetivo e direto que avalie a qualidade oocitária; por isso, são utilizados seus marcadores indiretos e, entre estes, a ida$\mathrm{de}^{9}$. Considera-se que a idade é marcador melhor que os niveis de FSH (hormônio folículo-estimulante) ${ }^{10,11}$, tanto para a obtenção de gestação ${ }^{8-11}$ quanto da taxa de implantação e aborto ${ }^{8,9}$. Estes parâmetros refletem, em última instância, a qualidade dos oócitos ${ }^{8-11}$. A Sociedade Européia de Reprodução Humana e Embriologia (ESHRE) também reconhece a idade como um marcador acurado para taxa de gestação em reprodução assistida ${ }^{4}$.

Entre os testes para avaliação da reserva ovariana, empregam-se os marcadores bioquímicos, os métodos de imagem e os testes dinâmicos ${ }^{4}$. Os marcadores bioquímicos empregados são: FSH basal, estradiol, inibina A e B e fator de inibição mülleriana ${ }^{12}$. Os marcadores de imagem são: contagem dos folículos antrais, volume ovariano total e análise do fluxo das artérias uterinas ${ }^{4,13}$. Os testes dinâmicos, por sua vez, são: teste após estímulo com clomifeno e a resposta de estradiol e inibina após estímulo com FSH ou agonista do GnRH (hormônio liberador de gonadotrofinas) ${ }^{4}$.

O mais empregado é o FSH basal, dosado do $2^{\circ}$ ao $4^{\circ}$ dia do ciclo $^{10,11}$. É, porém, considerado marcador de moderado valor para análise da res- posta ovariana em ciclos de reprodução assisti$\mathrm{da}^{4,8,10,11}$. A resposta ovariana é avaliada com maior acurácia com a contagem dos folículos antrais no $3^{\circ}$ dia do ciclo (presença de menos que 4 folículos antrais no total sugere má resposta ovariana) $)^{4,13}$.

O teste após estímulo com clomifeno acrescenta vantagem em termos de sensibilidade (de $6,6 \%$ somente para o FSH a $25,9 \%$ para o teste do clomifeno), mas não justifica o custo nem a exposição à droga ${ }^{4}$.

Portanto, a dosagem do FSH basal, e também a contagem antral folicular ${ }^{13}$, são os melhores marcadores de cancelamento de ciclo e número de oócitos que podem ser captados em reprodução assistida $^{9,11,14,15}$. A contagem de folículos antrais ainda mostra-se superior ao FSH basal quanto à análise de má resposta ovariana ${ }^{13}$. Porém, ambos são ruins como marcadores de sucesso do procedimento de reprodução assistida ou taxa de gestação, que está mais correlacionada com a idade ${ }^{4,9,11,13}$.

Desta forma, os testes para análise da reserva ovariana, quando isolados, não devem ser supervalorizados porque não são diagnósticos ${ }^{9,16}$. Ou seja, seu uso deve ser cauteloso e feito sempre conjuntamente com a idade, pois mulheres jovens FSH moderadamente elevado (10-20 UI/L) ainda apresentam taxas de gestação satisfatórias, apesar de apresentarem maior risco de cancelamento de ciclos de estimulação ovariana (para FSH $>5$ UI/L, a taxa de gestação é de $28 \%$ para mulheres jovens) ${ }^{11}$. Portanto, em mulheres jovens com má reserva ovariana, os oócitos existentes apresentam boa qualidade e se associam com adequadas taxas de gestação $0^{9,11}$.

Torna-se, então, mais adequada a análise conjunta destes dois fatores (idade e reserva ovariana) para a abordagem de mulheres submetidas à reprodução assistida ${ }^{11,16,17}$.

O objetivo deste trabalho foi avaliar a evolução das taxas de gestação de acordo com a idade em mulheres inférteis submetidas a procedimentos de reprodução assistida (FIV e injeção intracitoplasmática de espermatozóide - ICSI), atendidas em serviço terciário de infertilidade.

\section{Métodos}

Foi realizado estudo transversal retrospectivo, com análise de 1016 ciclos de hiperestimulação ovariana controlada (HOC) de 932 mulheres inférteis com idade entre 22 e 46 anos. Todas tinham indicação para FIV (370 ciclos) ou ICSI (646 ciclos) em serviço terciário para tratamento de casais inférteis. 
Todas as pacientes tinham indicação formal para realização de procedimentos de reprodução assistida de alta complexidade (anovulação crônica resistente a outras formas de tratamento, obstrução tubária e/ou fator masculino) e foram submetidas à propedêutica-padrão ${ }^{18}$ para investigação do casal infértil antes da realização do procedimento, composta por exames de citologia oncótica e swab endocervical para excluir infecções cervicais por bactérias aeróbias e pelos organismos Mycoplama hominis e Ureaplasma urealyticum, ultra-sonografia transvaginal, histerossalpingografia, espermograma com capacitação espermática, histeroscopia, dosagens sanguineas de TSH (hormônio tiroestimulante) e prolactina e sorologias para hepatite B e C, HIV, rubéola e sífilis.

A laparoscopia diagnóstica foi realizada nas mulheres cujo fator masculino não indicava isoladamente a realização do procedimento de reprodução assistida de alta complexidade (FIV ou ICSI). Em mulheres com suspeita de anovulação, foram solicitadas também as dosagens sanguíneas de FSH basal e LH (hormônio luteinizante), dosados no terceiro dia do ciclo e, naquelas com presença de hirsutismo, dosagens sanguíneas de sulfato de deidroepiandrosterona, testosterona total e 17hidroxiprogesterona.

Para análise da reserva ovariana, foi realizada dosagem de FSH basal ( $3^{\circ}$ dia do ciclo) em todas as pacientes com idade superior a 35 anos. Foram incluidas no estudo somente as pacientes que apresentavam niveis de FSH basal menores que $15 \mathrm{UI} /$ L ou teste do clomifeno menor que $18 \mathrm{UI} / \mathrm{L}$. Tal exame foi dispensado naquelas pacientes com menos de 35 anos e ciclos menstruais regulares.

Foram excluídas aquelas pacientes que iniciaram o ciclo de hiperestimulação ovariana, porém não foram submetidas à transferência embrionária por suspensão do ciclo (má resposta ovariana, cistos ovarianos, etc.) ou por ausência de embriões formados. Também foram excluídas aquelas pacientes que receberam doação de oócitos.

A principal variável analisada foi a taxa de gravidez (diagnóstico químico ou clínico) de acordo com a idade das pacientes no momento da realização do procedimento. Também foi avaliada a taxa de aborto nesta amostra.

\section{Protocolo de Reprodução Assistida (FIV ou ICSI)}

Inicialmente, foi realizada a programação do período menstrual com o uso de anticoncepcionais orais combinados no ciclo prévio àquele da indução da ovulação. Para dessensibilização hipofisária, foi utilizado o agonista do GnRH, acetato de leuprolida (Lupron ${ }^{\circledR}$, Abbott; Reliser ${ }^{\circledR}$, Serono, Brasil), (10 UI/ dia via subcutânea) ou nafarelina (via nasal 400 $\mu \mathrm{g} /$ dia) (Synarel ${ }^{\circledR}$, Pharmacia, Brasil). A dessensi- bilização hipofisária foi iniciada na fase lútea do ciclo prévio ou sete dias antes da menstruação programada. Do $1^{\circ}$ ao $3^{\circ}$ dia do ciclo de estimulação ovariana, foi realizada ultra-sonografia transvaginal (USTV) para excluir cistos ovarianos e então iniciada a estimulação ovariana controlada com gonadotrofinas de mulher menopausada: hMG (Menogon $^{\circledR}$, Ferring, Brasil; Pergonal ${ }^{\circledR}$, Serono, Brasil) ou FSH recombinante $\left(\right.$ Puregon $^{\circledR}$, Organon, Brasil; Gonal- ${ }^{\circledR}$, Serono, Brasil) nos primeiros 5 dias na dose de 200 a $450 \mathrm{UI} /$ dia e a partir do $6^{\circ}$ dia a dose da gonadotrofina foi ajustada de acordo com o crescimento folicular monitorizado por USTV.

Algumas pacientes usaram antagonista do GnRH (Cetrotide ${ }^{\circledR}$, Serono, Brasil) ao invés de agonista, com o objetivo de evitar picos prematuros de LH. Esta medicação foi iniciada quando o diâmetro médio dos folículos atingiu $14 \mathrm{~mm}$.

Quando houve pelo menos dois folículos com $17 \mathrm{~mm}$ de diâmetro médio, foi administrado gonadotrofina coriônica (hCG) intramuscular (Profasi ${ }^{\circledR}$, Serono, Brasil) 10.000 UI ou hCG recombinante 250 $\mu \mathrm{g}$ via subcutânea (Ovidrel ${ }^{\circledR}$, Serono, Brasil) e, após 34 a 36 horas, realizada a captação de oócitos por punção do fundo de saco vaginal guiada por USTV. O conteúdo de cada folículo foi então aspirado sob pressão de 120-130 mmHg em tubos de Falcon e mantido a $37^{\circ} \mathrm{C}$. Os complexos cumulus-oócitos foram então manuseados e separados do restante do fluido folicular em capela de fluxo de ar laminar para depois serem mantidos em meio de cultura em incubadora com temperatura controlada a $37^{\circ} \mathrm{C}$ e concentração de $\mathrm{CO}_{2}$ a $5 \%$. Após 2 a 5 horas em cultivo, os oócitos foram inseminados para FIV ou ICSI e os embriões formados foram mantidos em cultivo por mais 72 horas para serem, então, transferidos intraútero com guia de ultra-sonografia transabdominal.

A suplementação lútea foi iniciada a partir do dia da captação oocitária e, no caso de gravidez, até a $12^{\text {a }}$ semana de gestação. Os tipos de progesterona utilizados foram: diidrogesterona $30 \mathrm{mg} / \mathrm{dia}$ (Duphaston ${ }^{\circledR}$, Solvay, Brasil) ou a progesterona gel, uma aplicação diária (Crinone 8\%, Serono, Brasil).

Considerou-se gestação química a presença de teste de gravidez positivo por meio de $\beta$-hCG sérico (maior que $25 \mathrm{mUI} / \mathrm{mL}$ quatorze dias após transferência embrionária) medido por quimioluminescência, mas ausência de embrião à ultrasonografia. Considerou-se gestação clínica a presença de saco gestacional visivel ao exame ultrasonográfico quando realizado quatro a seis semanas após a transferência embrionária.

\section{Análise Estatistica}

Para análise estatística, os dados foram agrupados tomando-se como idade limite 30 anos. Um dos grupos incluiu todos os ciclos de $\mathrm{HOC}$ em 
mulheres com idade menor ou igual a 30 anos (2230 anos) e o outro grupo, aquelas com mais de 30 anos (31-46 anos). A seguir foi feita a comparação da taxa de gravidez entre estes dois grupos pelo teste do $\chi^{2}$. Foi utilizado o programa GraphPad Prism 3.0 para análise estatística e considerouse um nivel de significância de $5 \%(p<0,05)$. Foi incluída nos cálculos estatísticos tanto a gravidez química quanto a clínica.

O presente trabalho foi aprovado pelo Comitê de Ética da instituição onde foi realizado o estudo. O termo de consentimento foi lido e voluntariamente assinado pelas pacientes antes de realizar o procedimento de reprodução assistida.

\section{Resultados}

A taxa total de gravidez foi de $36,4 \%$ e as taxas de gravidez de acordo com cada faixa etária são apresentadas na Tabela 1 . Observou-se redução significativa na taxa média de gestação em mulheres com mais de 30 anos quando comparadas àquelas com idade inferior $(25,1$ e $45,4 \%$, respectivamente, para o grupo entre 31-46 anos e 22 aos 30 anos de idade, $p=0,0001$ ) (Figura 1).

Tabela 1 - Taxa de gravidez em porcentagem de acordo com cada faixa etária dos 22 aos 46 anos de idade em 1016 ciclos de hiperestimulação ovariana controlada (HOC) para FIV e ICSI.

\begin{tabular}{lccc}
\hline Idade & $\begin{array}{c}\text { Total de ciclos (HOC) } \\
\mathbf{n}\end{array}$ & $\begin{array}{c}\text { Gestações } \\
\mathbf{n}\end{array}$ & $\begin{array}{c}\text { Taxa de gravidez } \\
\%\end{array}$ \\
\hline $22-25$ & 46 & 20 & 43,5 \\
$26-30$ & 257 & 117 & 45,5 \\
$31-35$ & 393 & 145 & 36,9 \\
$36-40$ & 283 & 84 & 29,7 \\
$41-46$ & 37 & 11 & 10,8 \\
Total & 1016 & 377 & 37,1 \\
\hline
\end{tabular}

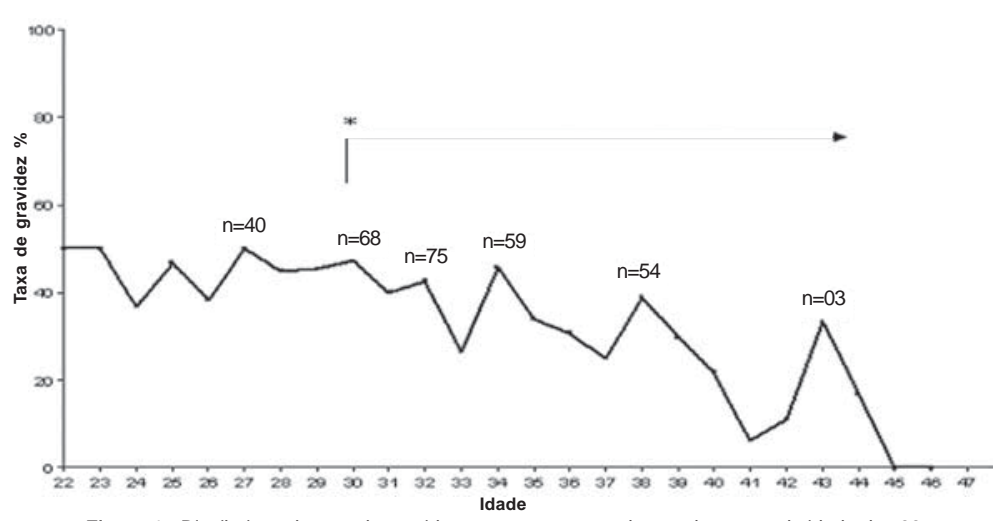

Figura 1 - Distribuição da taxa de gravidez em porcentagem de acordo com cada idade dos 22 aos 46 anos de idade em 1016 ciclos de hiperestimulação ovariana controlada para FIV e ICSI. Houve redução significativa da taxa de gravidez entre as mulheres com mais de 30 anos de idade (31-46). $n=$ número total de pacientes em determinada faixa etária. ${ }^{*} p<0,05$, teste do $\chi^{2}$.
Não foi observada diferença significativa na taxa de aborto entre os ciclos de HOC do grupo de mulheres com 22 a 30 anos (10,2\%) quando comparadas ao grupo com idade de 31 a 46 anos $(11,6 \%)(p=0,6854)$.

\section{Discussão}

Neste estudo observamos que a taxa de gravidez em 1016 ciclos de HOC de mulheres inférteis submetidas a procedimentos de reprodução assistida (FIV e ICSI) reduziu-se de forma significante a partir dos 30 anos.

Entretanto, não foi observada diferença significativa na taxa de aborto quando foram comparados os ciclos de mulheres com idade inferior a 30 anos (22 a 30 anos) e os ciclos referentes àquelas com mais de 30 anos (31 a 46 anos).

Existem evidências consistentes de que a fertilidade declina acentuadamente a partir dos 40 anos de idade ${ }^{2,4}$. A partir desta idade, apesar de ciclos regulares e níveis de estradiol e progesterona séricos normais, estas mulheres apresentam alteração da foliculogênese caracterizada pela ovulação a partir de um folículo de menor diâmetro do que as mais jovens, comprometendo o potencial oocitário de fertilização e implantação ${ }^{19}$. Acredita-se na existência de defeitos nos estágios tardios de desenvolvimento folicular antral possivelmente devidos a uma deficiência de fatores de crescimento como o IGF-I, IGF-II e LH ${ }^{19}$. Além disso, a ovulação em estágio precoce de desenvolvimento, com oócitos imaturos, compromete não apenas a qualidade oocitária como também reduz a probabilidade de fertilização, causando maior incidência de anomalias cromossômicas ${ }^{19}$. A integridade do fuso também parece se alterar com o aumento da idade ${ }^{4}$, contribuindo para a gênese das aneuploidias que são fortemente associadas com o aumento das taxas de aborto nas mulheres com idade avançada ${ }^{4}$.

A idade, portanto, compromete predominantemente a qualidade oocitária, e este fator supera o fator "quantidade oocitária", ou reserva ovariana, quando se consideram as taxas de implantação e gravide $z^{10,16}$. Por isso, em procedimentos de reprodução assistida, a idade é considerada o melhor indicador da taxa de gravide $z^{10,17}$.

Porém, estas alterações na qualidade oocitária serão significantes já a partir dos 30 anos de idade? Apesar de existirem relatos de redução da fertilidade feminina a partir de 30 anos ou até mesmo antes ${ }^{2,4}$, não há evidências consistentes de que alterações no microambiente folicular ou 
na qualidade oocitária ocorram até os 35 anos de idade $^{19}$. A primeira modificação endócrina ocorre por volta dos 35 anos que corresponderia à elevação dos niveis de FSH na fase folicular do ciclo devido à redução da produção de inibina B pelas células da granulosa, que, por sua vez, é ocasionada pela redução no pool oocitário ${ }^{2,19}$. Esta elevação poderia interferir na foliculogênese ${ }^{19}$. Os niveis de estradiol e progesterona não apresentam mudanças significativas por esta época ${ }^{1,2}$ e o endométrio também não representa fator limitante para a redução da fertilidade em mulheres a partir dos 30 anos de idade ${ }^{7}$. O número de folículos antrais, outro fator considerado importante para a avaliação do pool de folículos recrutáveis para a ovulação, também não mostra redução significativa até os 34 anos $^{2}$. Nesta idade observa-se média de 13,6 folículos antrais, ao passo que nas mulheres entre 41 e 46 anos a média é de $4,9^{2}$.

Portanto, não existem evidências de comprometimento da qualidade oocitária, da foliculogênese, da receptividade endometrial ou do eixo endócrino que indiquem que haja redução da fertilidade em mulheres com idade entre 30-35 $\operatorname{anos}^{1,2,19}$. A falta de um método que avalie diretamente a qualidade oocitária provavelmente limita o estudo adequado desta variável ${ }^{4,20}$. A biópsia embrionária pré-implantacional, bem como a análise da integridade do fuso ${ }^{4}$, representam métodos indiretos de avaliação da qualidade embrionária, e seu aprimoramento poderá trazer informações a respeito de alterações moleculares oocitárias que comprometam a sua qualidade e os resultados dos procedimentos de reprodução assistida ${ }^{4,20}$.

Quanto à avaliação da reserva folicular ovariana, o FSH basal, juntamente com a contagem dos folículos antrais, representam os melhores marcadores para o número de oócitos captados, má resposta ovariana e cancelamento de ciclos de hiperestimulação ovariana em procedimentos de reprodução assistida ${ }^{11,13,15,16}$ e devem ser usados para rastreamento de mulheres inférteis antes de serem submetidas a estes procedimentos de reprodução assistida. Contudo, a análise destes parâmetros deve sempre ser feita conjuntamente com a idade ${ }^{9-11,16}$. A dosagem do fator de inibição mülleriano, produzido pelos folículos pré-antrais e antrais, também emerge como método promissor para avaliação da reserva ovariana ${ }^{12}$.

Van Rooij et al. ${ }^{17}$ compararam dois grupos de mulheres em FIV: aquelas com mais de 40 anos e niveis de FSH menores que $15 \mathrm{UI} / \mathrm{L}$ e mulheres jovens com valores de FSH superiores a $15 \mathrm{UI} / \mathrm{L}$ e observaram que mulheres jovens, mesmo com niveis de FSH elevados, apresentaram porcentagem mais elevada de cancelamento dos ciclos de estimulação ovariana, porém, com taxas mais altas de implantação por embrião transferido e de gestação por ciclo.

Em outro estudo ${ }^{16}$ foram incluídas $118 \mathrm{mu}$ lheres com idade menor que 38 anos e niveis de FSH superiores a $10 \mathrm{UI} / \mathrm{L}$ que foram comparadas com 577 mulheres com mais de 38 anos e FSH menor que $10 \mathrm{UI} / \mathrm{L}$. As taxas de nascidos vivos foi maior no grupo de mulheres mais jovens $(21 \%$ de nascidos vivos em relação a $12 \%$ nas mulheres mais velhas) ${ }^{16}$.

Estes achados comprovam o fato de que a idade ainda é o melhor marcador para taxa de gestação em reprodução assistida e indica, em últi-

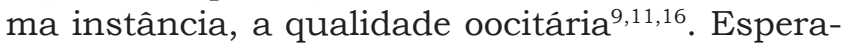
se que a melhora da compreensão da genética oocitária e da maneira como contribui para a viabilidade embrionária possa fornecer novos indicadores que sejam capazes de avaliar diretamente a qualidade oocitária ${ }^{4}$. Além disso, espera-se que os médicos ginecologistas reconheçam esta limitação da idade para o sucesso dos procedimentos de reprodução assistida e, desta forma, não posterguem o diagnóstico e o tratamento da infertilidade.

\section{Referências}

1. van Rooij IA, Bancsi LF, Broekmans FJ, Looman CW, Habbema JD, te Velde ER. Women older than 40 years of age and those with elevated folliclestimulating hormone levels differ in poor response rate and embryo quality in in vitro fertilization. Fert Steril. 2003;79(3):482-8.

2. van Zonneveld P, Scheffer GJ, Broekmans FJ, Blankenstein MA, de Jong FH, Looman CW, et al. Do cycle disturbances explain the age-related decline of female fertility? Cycle characteristics of women aged over the 40 years with a reference population of young women. Hum Reprod. 2003;18(3):495-501.

3. Badalotti $\mathrm{M}$, Petracco A. Idade e fertilidade. In: Badalotti M, Teloken C, Petracco A, editores. Fertilidade e infertilidade humana. Rio de Janeiro: MEDSI; 1997. p.101-13.

4. Baird DT, Collins J, Egozcue J, Eyers LH, Gianaroli L, Leridon H, et al. Fertility and ageing. Hum Reprod Update. 2005;11(3):261-76.

5. Toner JP, Flood JT. Fertility after the age of 40 . Obstet Gynecol Clin North Am. 1993;20(2):261-72.

6. Navot D, Bergh PA, Williams MA, Garrisi GJ, Guzman I, Sandler B, et al. Poor oocyte quality rather than implantation failure as a cause of agerelated decline in female fertility. Lancet. 1991;337(8754):1375-7. 
7. Navot D, Drews MR, Bergh PA, Guzman I, Karstaedt A, Scott RT Jr, et al. Age-related decline in female fertility is not due to diminished capacity of the uterus to sustain embryo implantation. Fertil Steril. 1994;61(1):97-101.

8. Toner JP. Ovarian reserve, female age and the chance for successful pregnancy. Minerva Ginecol. 2003;55(5):399-406.

9. Toner JP. Modest follicle-stimulating hormone elevations in younger women: warn but don't disqualify. Fertil Steril. 2004;81(6):1493-5.

10.van Rooij IA, de Jong E, Broekmans FJ, Looman CW, Habbema JD, te Velde ER. High folliclestimulating hormone levels should not necessarily lead to the exclusion of subfertile patients from treatment. Fertil Steril. 2004;81(6):1478-85.

11. Toner JP. Age = egg quality, FSH level = egg quantity . Fertil Steril. 2003;79(3):491.

12. Silberstein T, MacLaughlin DT, Shai T, Trimarchi JR, Lambert-Messerlian G, Seifer DB, et al. Mullerian inhibiting substance levels at the time of HCG administration in IVF cycles predict both ovarian reserve and embryo morphology. Hum Reprod. 2006;21(1):159-63.

13. Hendriks DJ, Mol BW, Bancsi LF, Te Velde ER, Broekmans FJ. Antral follicle count in the prediction of poor ovarian response and pregnancy after in vitro fertilization: a meta-analysis and comparison with basal follicle-stimulating hormone level. Fertil Steril. 2005;83(2):291-301.
14. Weghofer A, Margreiter M, Fauster Y, Schaetz T, Brandstetter A, Boehm D, et al. Age-specific FSH levels as a tool for appropriate patient counselling in assisted reproduction. Hum Reprod. 2005;20(9):2448-52.

15. Roberts JE, Spandorfer S, Fasouliotis SJ, Kashyap $\mathrm{S}$, Rosenwaks $Z$. Taking a basal follicle-stimulating hormone history is essential before initiating in vitro fertilization. Fertil Steril. 2005;83(1):37-41.

16. Abdalla H, Thum MY. An elevated basal FSH reflects a quantitative rather than qualitative decline of the ovarian reserve. Hum Reprod. 2004;19(4):893-8.

17.van Rooij IA, de Jong E, Broekmans FJ, Looman CW, Habbema JD, te Velde ER. The limited value of follicle-stimulating hormone as a test for ovarian reserve. Fertil Steril. 2004;81(6):1496-7.

18. Ferriani RA, Navarro PAAS. Protocolos de condutas em infertilidade conjugal. São Paulo: Conexão Brasil; 2004.

19. Santoro N, Isaac B, Neal-Perry G, Adel T, Weingart L, Nussbaum A, et al. Impaired folliculogenesis and ovulation in older reproductive aged women. J Clin Endocrinol Metab. 2003;88(11):5502-9.

20. Combelles CM, Racowsky C. Assessment and optimization of oocyte quality during assisted reproductive technology treatment. Semin Reprod Med. 2005;23(3):277-84. 